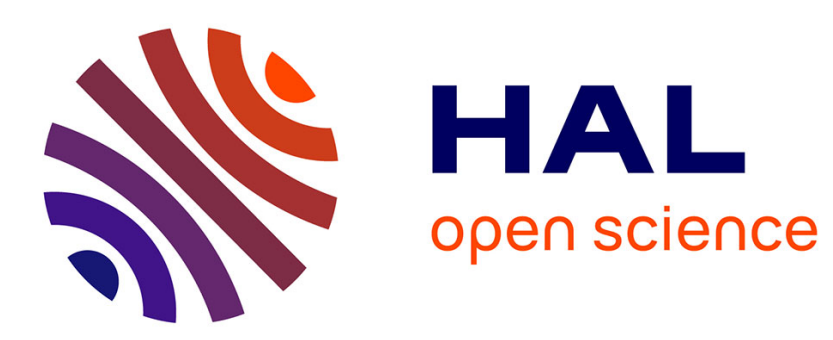

\title{
Direct Noise Prediction and Control of an Installed Large Low-speed Radial Fan
}

\author{
Marlène Sanjosé, Stéphane Moreau
}

\section{To cite this version:}

Marlène Sanjosé, Stéphane Moreau. Direct Noise Prediction and Control of an Installed Large Lowspeed Radial Fan. 16th International Symposium on Transport Phenomena and Dynamics of Rotating Machinery, Apr 2016, Honolulu, United States. hal-01887481

\section{HAL Id: hal-01887481 \\ https://hal.science/hal-01887481}

Submitted on 4 Oct 2018

HAL is a multi-disciplinary open access archive for the deposit and dissemination of scientific research documents, whether they are published or not. The documents may come from teaching and research institutions in France or abroad, or from public or private research centers.
L'archive ouverte pluridisciplinaire HAL, est destinée au dépôt et à la diffusion de documents scientifiques de niveau recherche, publiés ou non, émanant des établissements d'enseignement et de recherche français ou étrangers, des laboratoires publics ou privés. 


\title{
Direct Noise Prediction and Control of an Installed Large Low-speed Radial Fan
}

\author{
Marlène Sanjose $^{1 *}$, Stéphane Moreau ${ }^{1}$
}

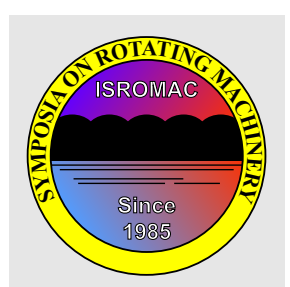

ISROMAC 2016

International

Symposium on Transport

Phenomena and Dynamics of

Rotating Machinery

Hawaii, Honolulu

April 10-15, 2016

\section{Abstract}

The aeroacoustics of several configurations of a large complex radial fan module typical of ventilation systems used in building or large transportation systems has been directly simulated with a Lattice Boltzmann Method. For the reference configuration the available flow measurements are well captured by the simulation and excellent noise predictions are achieved for all microphones both in terms of levels and spectral shape. The inlet distortion and a flow separation at the fan blade foot are shown to cause the observed strong tonal noise at the blade passing frequency. The unsteadiness of the tonal noise is also traced to large "cucumber-like" coherent structures wandering on the fan hub and hitting the blade leading edge quite randomly. Fan hub contouring is shown to almost eliminate the flow detachment at the hub and improve fan performances. Yet it does not reduce the inlet distorsion and has only a marginal effect on the noise. Adding a thin filter on the bellmouth removes the large turbulent structures ingested by the fan and improves the flow-field uniformity at the fan inlet. Significant noise reduction is then achieved eventhough additional pressure losses are introduced. A final tonal noise control with a rotating obstruction placed on the fan bellmouth has been succesfully simulated yielding the expected sound modulation. All these flow and acoustic features compare remarkably well between the measurements and the simulations.

\section{Keywords}

Aeroacoustics - Radial fan — ventilation noise

${ }^{1}$ Université de Sherbrooke, Département de Génie Mécanique, Sherbrooke, J1K2R1, QC, Canada

*Corresponding author: stephane.moreau@usherbrooke.ca

\section{INTRODUCTION}

Low-speed radial fans are used in many residential applications for ventilation, cooling and heating systems. The noise level produced by these systems is one of the main concerns for customers. Thus, aeroacoustic predictions are required by the manufacturers at the design level. Aero-acoustic sources are multiple in complex systems. Broadband noise component is produced by a wide range of turbulent excitations while tonal noise component is rather related to large coherent flow structures strongly depending on the installation. In the present work, unsteady compressible numerical simulations are used to predict tonal and broadband noise components of the acoustic spectra for a radial low-speed fan installed in a complex module typical of many ventilation systems in transportation applications. Very few numerical tools allow capturing at affordable computational cost, both the tonal and broadband sources and the far-field propagation as it requires to capture large structures related to the installation to very small turbulent structures that appear in boundary layers, and a wide range of wave-numbers on a large computational domain $[1,2]$. Even fewer involve the complete installation system around the fan. The present methodology relies on direct noise generation and propagation using the LatticeBoltzmann Method (LBM) on the complete installation and the fast turnaround of the simulations allow testing several passive or semi-active control strategies. The former involves fan hub contouring and adding a simple flow straightener. The latter consists of flow obtructions placed upstream of the fan, the Simple Silence technology ${ }^{\mathrm{TM}}$ developed at Université de Sherbrooke (UdeS). These simulations are compared and validated with experiments run in the acoustic facilities at UdeS.

The present experimental and numerical methodology is described in the next section. The results on the baseline and modified configurations are presented in the following sections. Conclusions on the accuracy of the numerical method and the noise control strategies are finally drawn.

\section{METHODOLOGY}

\subsection{Experimental mock-up and test set-up}

The investigated large radial fan has a $50 \mathrm{~cm}$ diameter, a $40 \mathrm{~cm}$ height and is typical of ventilation systems used in building or large transportation systems (railroad application for instance). It is installed in a large module as presented in Fig. 1(a). The latter involves a large plenum with several inlets and a stack of heat exchangers connected at the outlet to two rectangular ducts with varying sections. The rotational speed is 1200 rpm yielding a low maximum Mach number of 0.05 . The plenum is connected to the system circular duct through an elliptic adapter. The whole module has been tested in the semianechoic room at UdeS as shown in Fig. 1(b), where volume flow-rates in the two outlet ducts were measured simultaneously with sound pressure levels in the far-field. The flow rates have been estimated following the ISO-3966 standard, e.g. the velocity has been measured along several lines at the outlet ducts with a Pitot tube. The far-field acoustic pressure has been measured at several locations around the mock-up with a B\&K microphone to yield the module directivity, but 

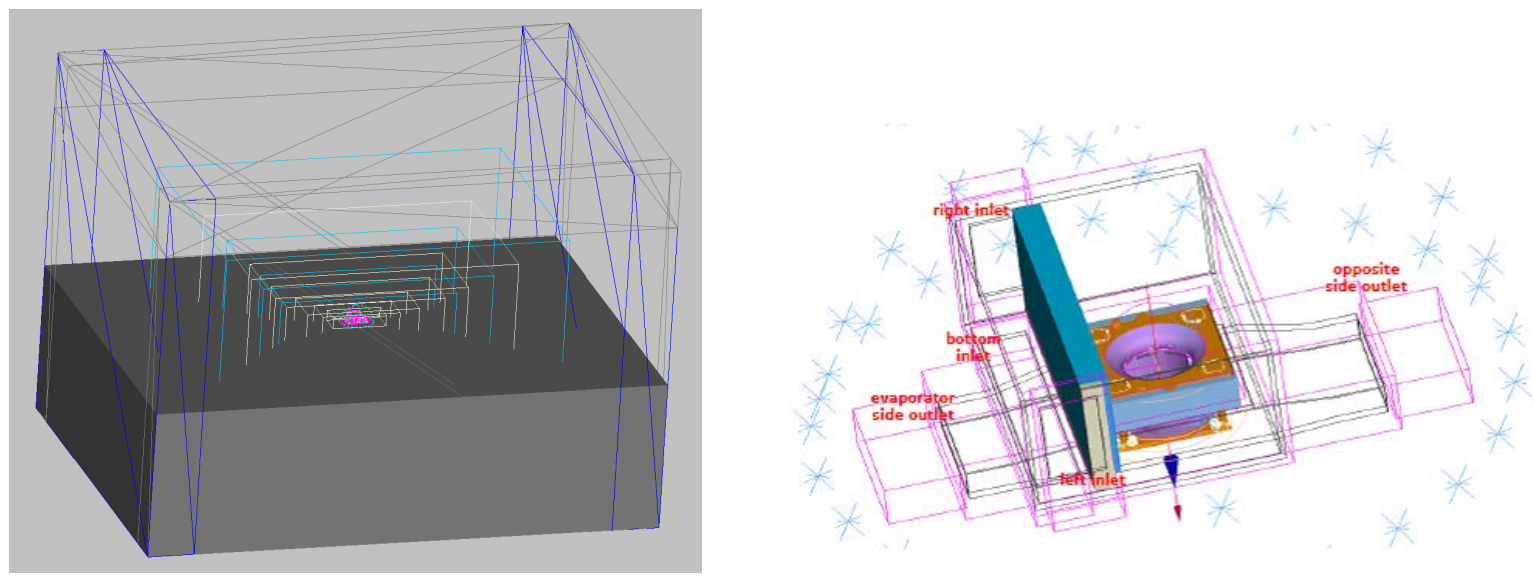

(a) Numerical investigated configuration and set-up: (left) large anechoic computational domain with all refinement regions, the module in the middle and the rigid ground; (right) isometric view of the full cooling module with all the probes around it where the acoustic pressure has been collected.

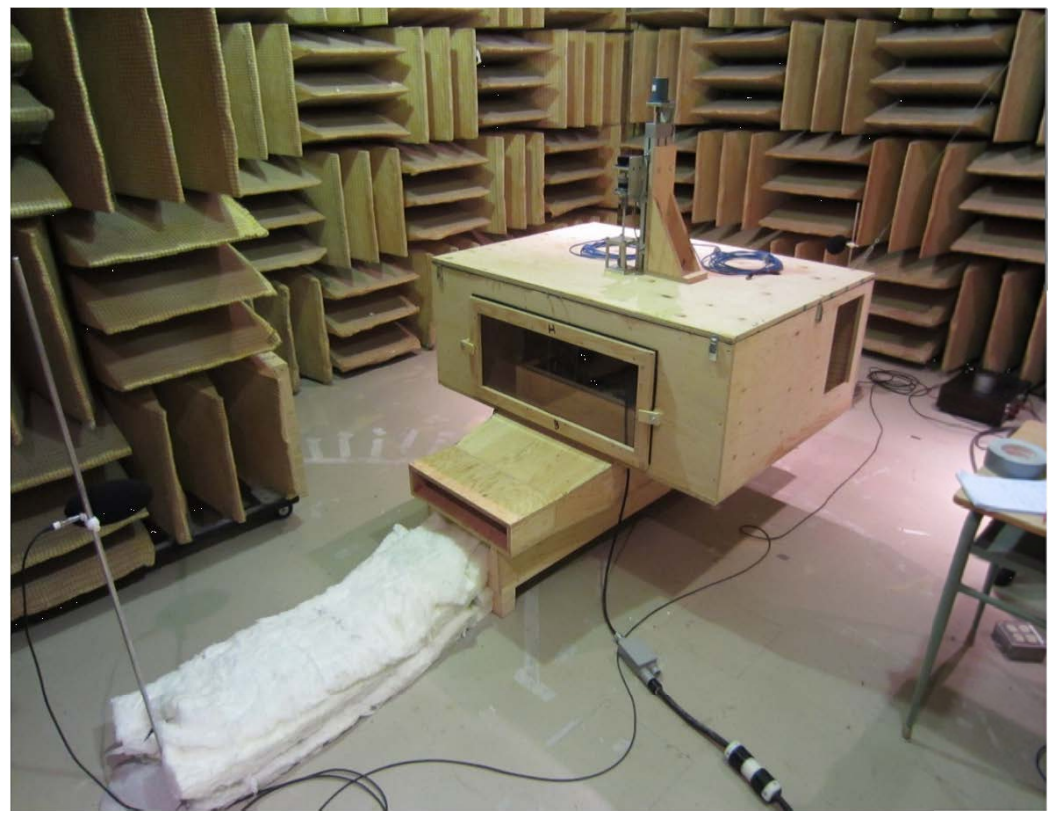

(b) Experimental configuration in the UdeS semi-anechoic room.

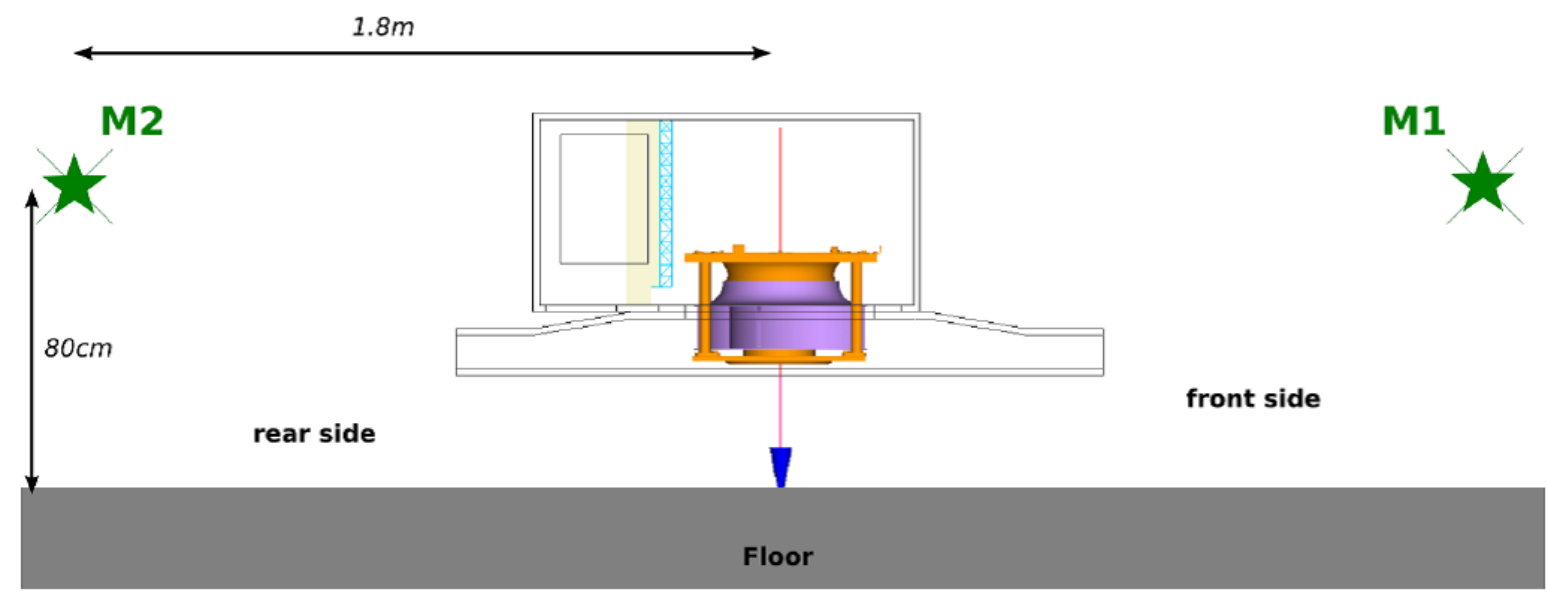

(c) Reference microphone positions M1 and M2 with respect to the module.

Figure 1. Installed low-speed radial fan investigated in the present study. 
the present study will mostly focus on two positions M1 and M2 above the outlet sections as shown in Fig. 1(c).

\subsection{Numerical set-ups and parameters}

The computational domain exactly reproduces the actual UdeS semi-anechoic room with a rigid ground. The flow recirculation in the room is therefore properly mimicked. The computational domain is meshed using cubic voxels only, with several voxel refinement (VR) zones, some of them are highlighted in pink and yellow in Fig. 1(a). Additional refinement zones are added outside the testing region to properly damp the acoustic field and reproduce the room anechoicity. Several mesh resolutions especially near the rotor have been investigated leading to a minimum voxel size of $1 \mathrm{~mm}$ and a final mesh of 88 million voxels, which provides grid-independent acoustic solutions on the baseline configuration. The Exa Powerflow 4.4 solver is used to perform the compressible Very Large Eddy Simulation (VLES) of this configuration. The interface between the rotor part and the stationary part of the domain is a sliding mesh interface as explained in [2].

The time step is selected by the solver to ensure a CFL number based on the speed of sound of one in the smallest voxels. This leads to a time-step of $1.65 \times 10^{-6} \mathrm{~s}$. The torque and the flow-rate are monitored during the simulation to evaluate the convergence of the system towards a steady state. Steady flow-rates on the inlets and outlets of the fan system were obtained after 2 fan revolutions. In addition to the two microphone probes M1 and M2 the static pressure at different probe locations (blue crosses in Fig. 1(a)) around the mockup are recorded to extract sound directivity. Unsteady pressures were recorded for at least $0.8 \mathrm{~s}$ to obtain a well resolved spectrum down to $100 \mathrm{~Hz}$. The same frequency resolution of $2.3 \mathrm{~Hz}$ is also used for all the shown spectra.

In addition to the baseline case, several passive noise control devices have also been simulated and tested. First a geometrical modification of the hub has been suggested based on the airflow analysis below. The latter has also suggested a better flow-conditioning at the fan inlet, which led to the addition of a filter on top of the fan system. The porosity of this filter has been experimentally determined in the UdeS laboratories: the viscous and inertial resistances are $113 \mathrm{~s}^{-1}$ and $87 \mathrm{~m}^{-1}$ respectively. Finally an obstruction has been added below the filter to provide tonal noise control [3, 4]. Based on previous tests, an optimal lobe mean diameter of $35 \mathrm{~cm}$ with an amplitude of $1.5 \mathrm{~cm}$ was selected. A minimal $3 \mathrm{~mm}$ space is imposed between the rotating domain and the non-rotating filter and convergent. All the numerically tested configurations are summarized in Table 1.

\section{RESULTS AND DISCUSSION}

\subsection{Baseline configuration}

The global performances of the installed fan are compared with the experimental measurements in Table 2. The flow distribution between the two sides is well represented but the numerical model underpredicts the experimental estimate by $12 \%$. Fig. 2 shows that this discrepancy is mostly caused by
Table 1. Summary of calculations.

\begin{tabular}{lc}
\hline Calculation Name & Geometry details \\
\hline Baseline & Initial setup \\
\hline New-Hub & Modified hub \\
\hline Filter & Initial setup with filter \\
\hline Filt-Obstr & $\begin{array}{c}\text { Initial setup with filter } \\
\text { and flow obstruction }\end{array}$ \\
\hline
\end{tabular}

the boundary layer resolution on the upper and lower wall of the ducts. They are numerically overestimated because of a lack of local grid resolution. Yet as the velocity distributions in the duct core are well predicted no further grid refinement was achieved to limit the model size.

Table 2. Comparison between measured and computed volume flow rates at the mock-up outlets.

\begin{tabular}{l|c|c|c} 
& Experiments & Simulation & Difference \\
\hline Evaporator side & $0.50 \mathrm{~m}^{3} / \mathrm{s}$ & $0.44 \mathrm{~m}^{3} / \mathrm{s}$ & $12 \%$ \\
\hline Opposite side & $0.48 \mathrm{~m}^{3} / \mathrm{s}$ & $0.42 \mathrm{~m}^{3} / \mathrm{s}$ & $12.5 \%$
\end{tabular}

The overall flow structure within the module is shown in Fig. 3. The particular geometry of the inlet chamber generates large distortions ingested by the fan. The incoming flows coming from the threefold inlets generate turbulent structures caused by their mixing that can be identified by the $\Lambda_{2}$ criterion in Fig. 3(a). The flow goes through the evaporator modeled by a porous medium that yields a uniform velocity profile along the whole width of the inlet box. Even if this side entrance is homogeneous, the strong fan suction generates a strong distorted flow pattern at the inlet of the fan as can be seen in Figs. 3(a) and 3(b). The fluid closest to the fan goes directly into the convergent while the more remote lines have to go all around the inlet box to finally enter the fan. These variations in flow paths generate strong distortions upstream of the fan. Such distortions are typical sources of tonal noise. A first control device to be introduced is therefore to limit this strong distortion and straighten the flow at the inlet with a filter.

Looking closer to the fan, a flow detachment also appears close to the motor and hub. This flow detachment is strongly unstable during the fan rotation and the position of the stagnation point on the top part of the motor varies slightly over time. These two observations have triggered a second control device: a more streamlined hub shape.

Acoustic results on the baseline configuration are shown for the two microphones M1 and M2 in Fig. 4 where "PF" stands for Powerflow predictions. The acoustic spectra computed from the static pressure history recorded by the probes shown in Fig. 1(c) are compared with acoustic measurements in Fig. 4. The numerical spectra agree very well with the ex- 

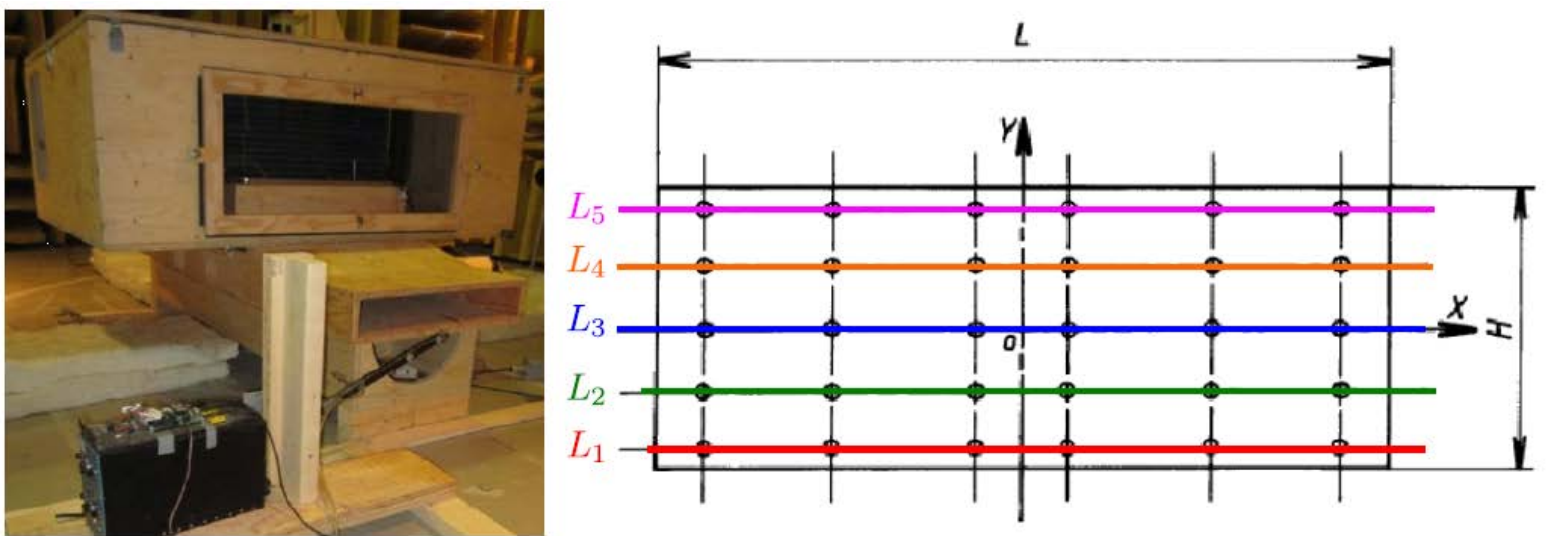

(a) Experimental set-up (left) and measurement grid (right) for the velocity measurements over the outlet sections.
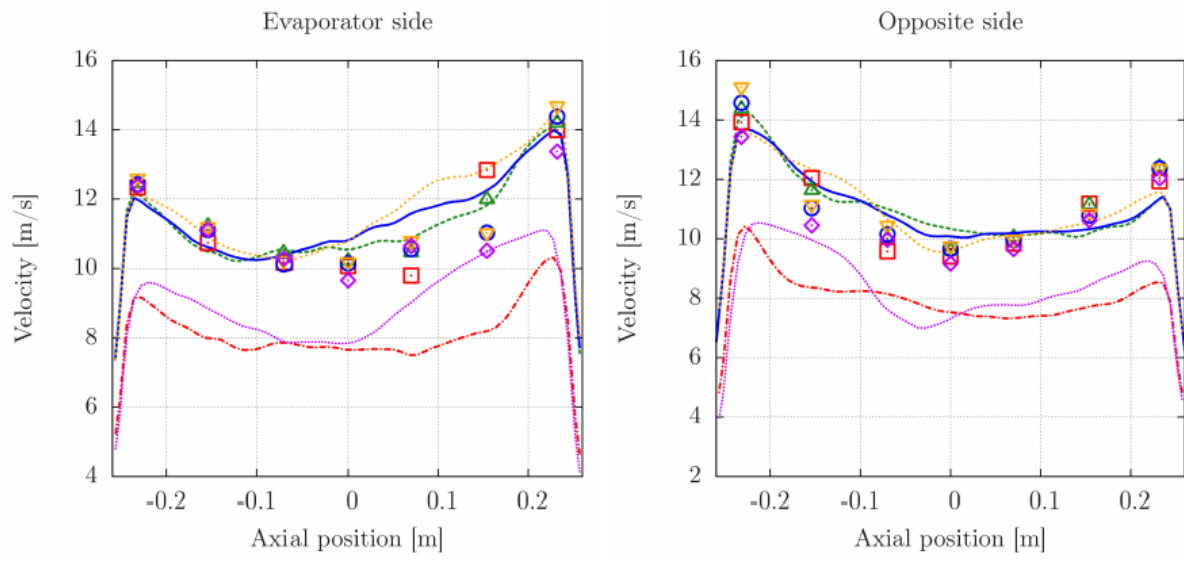

L1-num L2-num ............ L3-num L4-num

L1-exp ๑

L1-exp

L3-exp

L3-exp

L5-exp

(b) Velocity distribution at the mock-up outlets from experiment and the Baseline simulations.

Figure 2. Global performance comparisons.
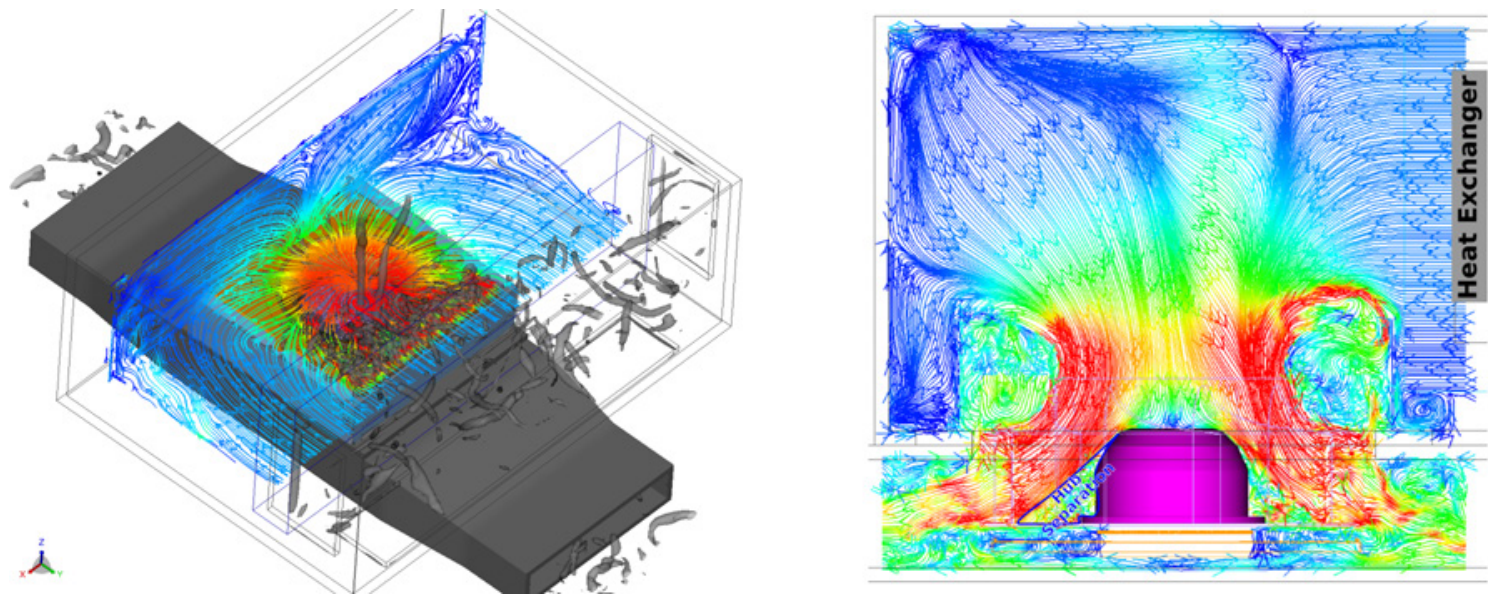

Figure 3. Reference configuration: module flow structure; (a) Iso contours of $\lambda_{2}$ factor combined with instantaneous streamlines colored by velocity magnitude; (b) Instantaneous streamlines colored by velocity magnitude. 

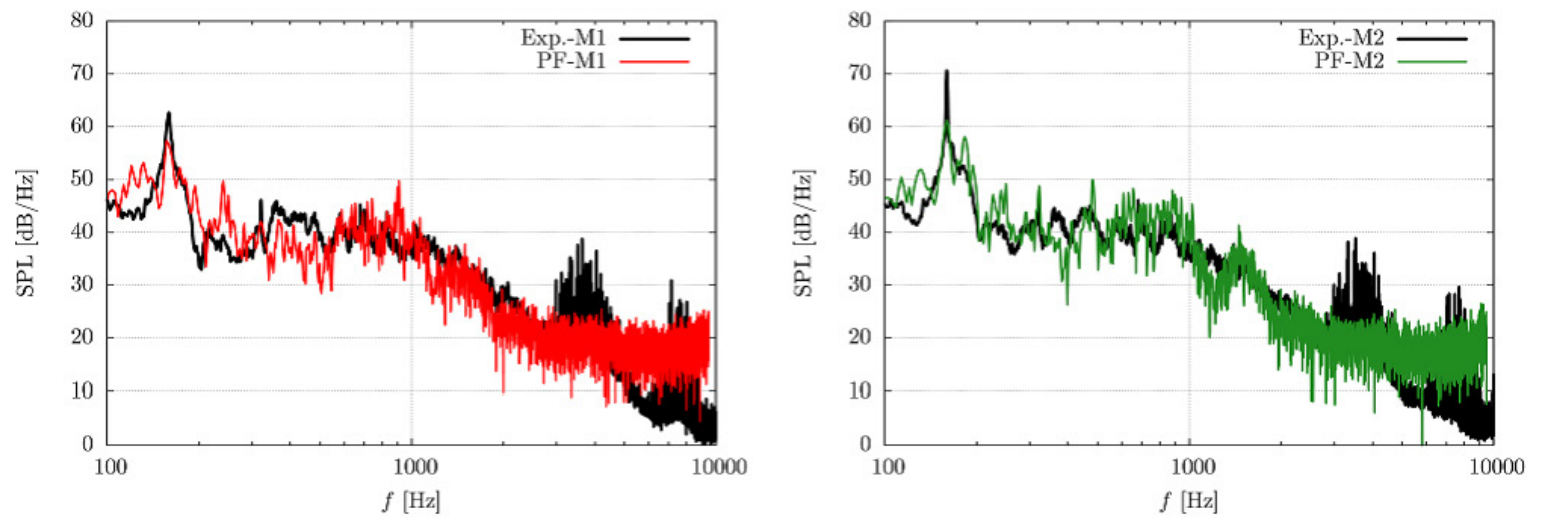

Figure 4. Reference configuration: acoustic comparisons on microphones M1 and M2.

perimental spectra up to $3000 \mathrm{~Hz}$. The comb of tones between $3000 \mathrm{~Hz}$ and $5000 \mathrm{~Hz}$ that appears in the experiments is related to the power supply converter from 50 to $60 \mathrm{~Hz}$. Above $5000 \mathrm{~Hz}$ the numerical errors limit the decay at high frequencies. The very good agreement of the spectrum shape at low frequency proves that the installation effects of the mock-up are well reproduced in the numerical setup. Even the observed fluctuations of the BPF tone are captured nicely. They are estimated using the standard deviation $\sigma$ of the Hilbert transform of the filtered signal around the BPF. The values for both the experiments and the numerical simulations are reported in Tab. 3. The integrated tone level is within $5 \mathrm{~dB}$ accuracy. The numerical simulation captures the instability fluctuation levels accurately.

Table 3. Tone peak and fluctuation amplitudes in $\mathrm{dB}$ for the Baseline configuration.

\begin{tabular}{l|l|ll|l} 
Case & Mean & Mean $+\sigma$ & Mean $-\sigma$ & diff \\
\hline Exp-M1 & 73.4 & 67 & 77 & 10 \\
Exp-M2 & 78.2 & 74 & 81 & 7 \\
\hline PF-M1 & 70.4 & 63.9 & 74.1 & 10.2 \\
PF-M2 & 74.0 & 68.1 & 77.5 & 9.4
\end{tabular}

A hint at the acoustic sources is provided by the zones of intense pressure fluctuations. The band-filtered power spectral density (PSD) of the wall-pressure fluctuations in the dominant frequency band of Fig. 4 is shown in Fig. 5. The highest pressure fluctuation levels are localized at the top of the blade leading-edges and may be related to the inhomogeneous incoming flow and the large turbulent eddies ingested by the fan. An additional source can be identified at the bottom of the blade leading-edges and may be related to the impact of the large turbulent eddies formed at the hub due to the flow detachment on the motor.

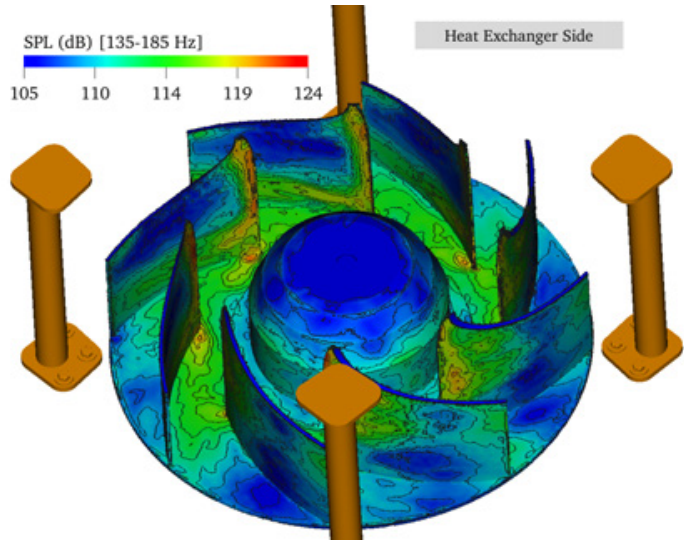

Figure 5. Reference configuration: band filtered power spectral density of the wall-pressure fluctuations.

\subsection{Modified hub configuration}

The unsteadiness on the fan blades is first related to the flow in the fan bowl. The shape of the motor was then modified to follow the streamlines found in the reference calculation. Its shape was however adapted to fit on the actual motor with thickness limitations of the plastic material and limitations of the actual bowl radius. The simulation setup including the modified hub geometry has been run as a continuation of the reference setup run. As the flow in the mock-up is already established, the transient time is significantly reduced from two rotation periods to only half one. The overall performances (torque and mass flow rates) are slightly improved as the torque is slightly reduced and a better flow-rate distribution is observed at the two duct outlets.

The influence of the modified hub on the flow patterns in the fan bowl is shown in Fig. 6. The flow near the motor is strongly improved, the massive detachment is reduced closer to the hub. The streamlines on the evaporator side are better aligned than in the Baseline case, but a larger unsteadiness appears on the opposite side. Also it must be highlighted that the flow in the entrance box is modified by the hub, leading to a flow angle slightly deviated when comparing with the 


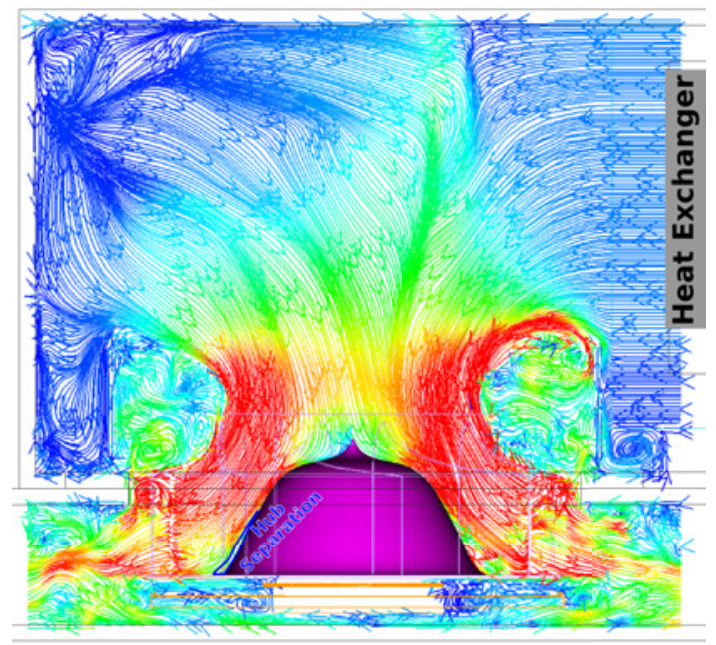

Figure 6. Modified hub configuration: flow topology (instantaneous streamlines colored by velocity magnitude).

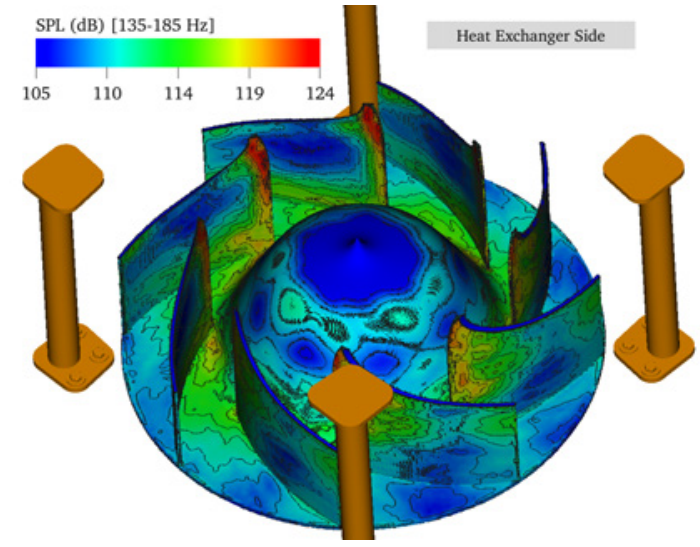

Figure 7. Modified hub configuration: band filtered power spectral density of the wall-pressure fluctuations.

Baseline case in Fig. 3(b). The hub geometry not only modifies the flow in the bowl but also the flow in the entrance box. Improving the flow in the fan itself (as can be seen by the torque reduction) degrades the inflow condition, increasing the variations of the angle of attack on the blade upper part of the leading-edge. These effects modify the blade response to the incoming turbulent flow as can be seen by the band-filtered PSD of wall-pressure fluctuations in Fig. 7. The various spots of high amplitude pressure fluctuations are decreased except the one at the leading edge of the blade, which is now more intense for the investigated frequency band.

The acoustic spectra from the pressure recorded at the experimental probe locations for the New-Hub case are compared with the Baseline case in Fig. 8. Very similar results are obtained for the New-Hub simulation showing the very low effect on the noise spectra of the flow detachment unsteadiness at the hub. As a consequence of the modification in inlet flow distorsion that has been highlighted in Figs. 6 and 7, the noise levels at the BPF have increased. The integrated level of the BPF are shown in Tab. 4. Despite higher in the PSD, the integration leads to a lower mean value in the New-Hub simulation than in the Baseline simulation. Similarly to previous section, the standard deviation $\sigma$ of the tone fluctuations is estimated using the Hilbert transform analysis. The range of fluctuations of the tone are of the same order than in the Baseline case, and even decreased on M1, the opposite side of the evaporator. This is a consequence of the improvement of the flow entrance coming from the evaporator side shown in Fig.6, that is then directed on the opposite side. A similar modification was prototyped (the electrical motor prevented from using the numerical optimal shape) and tested. As in the simulations the overall noise was slightly increased with even larger unsteadiness of the BPF.

Table 4. Tone peak and fluctuation amplitudes in $\mathrm{dB}$ for the New-Hub configuration.

\begin{tabular}{l|l|ll|l} 
Case & Mean & Mean $+\sigma$ & Mean $-\sigma$ & diff \\
\hline Exp-M1 & 75.1 & 68.3 & 78.9 & 10.6 \\
Exp-M2 & 79.1 & 74.8 & 81.9 & 7.1 \\
\hline PF-M1 & 73.0 & 68.6 & 75.9 & 7.3 \\
PF-M2 & 74.4 & 68.5 & 77.9 & 9.4
\end{tabular}

In summary, while decreasing the flow detachment at the hub, the modification of the hub geometry did not bring a significant noise reduction and a satisfactory stabilization of the BPF tone. Yet, it has shown the key influence of the flow quality at the fan inlet. Therefore as a second step in this analysis, the effect of a filter at the fan entrance is therefore investigated within the Filter simulation.

\subsection{Filter configuration}

When adding the porous medium mimicking the filter, an additionnal loss appears in the mock-up. As a consequence the flow-rate in the ducts are significantly decreased in the Filter simulation compared with the Baseline simulation (about 20\%).

Yet the filter modeled by a porous medium forces the flow streamlines to be parallel to the fan axis as can be seen in Fig. 9. Indeed, even though the effect of the asymmetric inlet box can be still detected in the streamline slopes above the filter, both sides of the fan are now equally fed by the flow, resulting in quite symmetric flow patterns in the fan after the filter.

The filter also decreases the tip-gap flow recirculation and avoids the large inlet turbulent structures (shown in Fig. 3(a) on the evaporator side) to be ingested by the fan as can been seen in Fig. 10. The flow in the upper part of the blade passage is consequently more uniform than in the Baseline simulation in Fig. 3(a). The flow rate is decreased due to the additional pressure loss created by the filter. It modifies the flow incidence at the blade leading edge and creates a stronger flow detachment. The many structures near the hub are created by the flow detachment and are similar to the Baseline simulation because the initial hub was used in the 

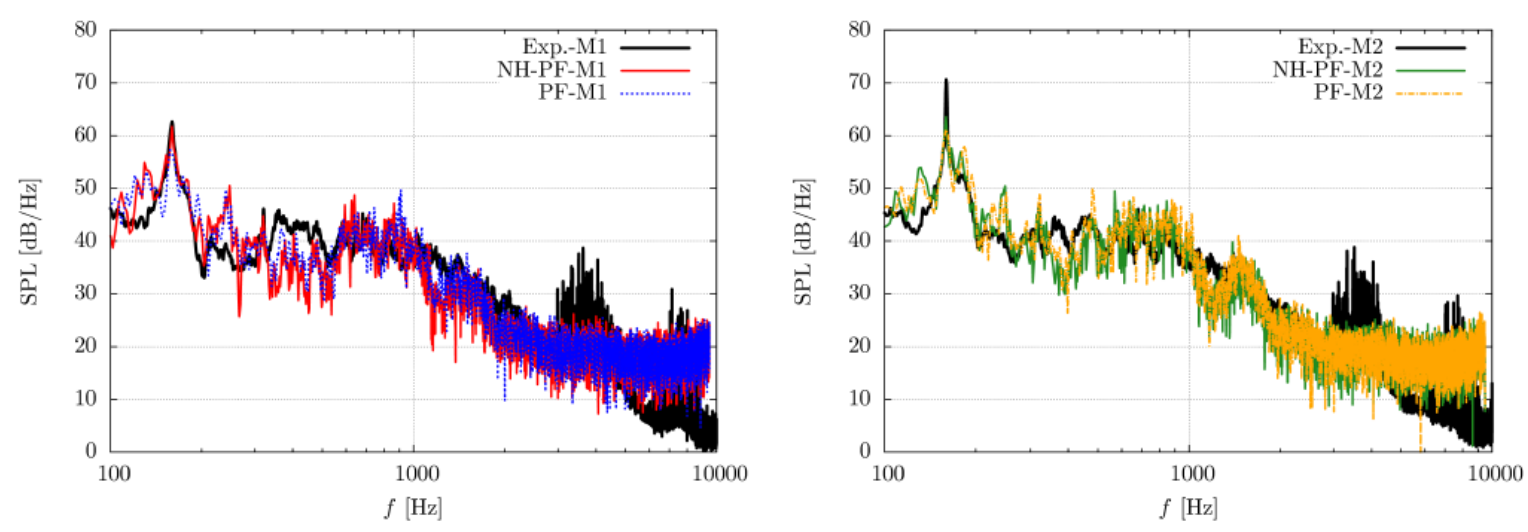

Figure 8. Modified hub configuration: acoustic comparisons on microphones M1 and M2.

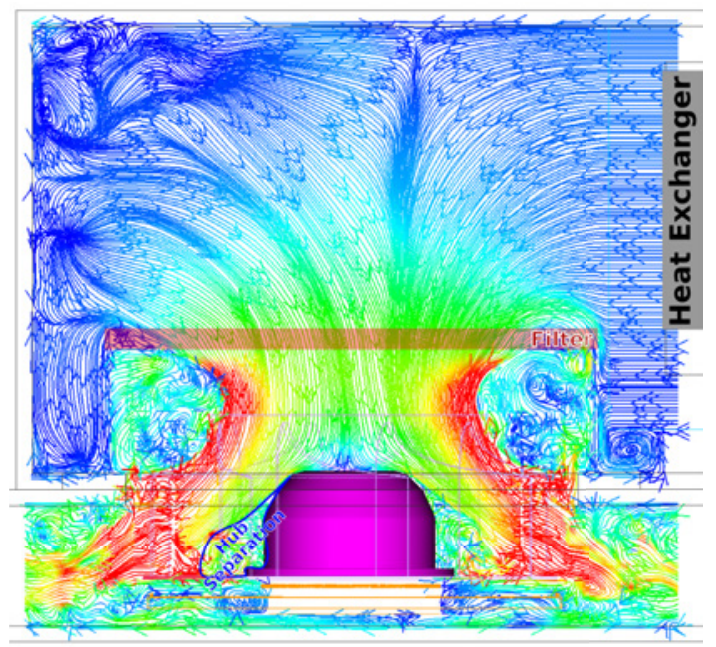

Figure 9. Filter configuration: flow topology (instantaneous streamlines colored by velocity magnitude; red box: filter).

\section{Filter simulation.}

The relative velocity contours at the upper part of the blade passages also show that the filter now brings a very regular and axisymmetric flow in the fan. Since the flow on the blade upper part is cleaner in the Filter setup, the wallpressure fluctuations at the BPF are strongly reduced as can be seen in Fig. 11. Additional high fluctuation levels appear at mid-chord of the blade and are possibly related to the flow detachment that appears in Fig. 10.

The far-field acoustic pressure is recorded similarly to the previous cases at the experimental probe locations shown in Fig. 1. The acoustic spectra from the Filter simulation are compared with those obtained in the Baseline simulation in Fig. 12. The filter provides a sound level reduction in the $[100-1500] \mathrm{Hz}$ range. This reduction is the result of several causes. First the flow rate is lower in the fan, reducing the steady and the unsteady loads on the blades. Also, the filter modifies the turbulent length scales ingested in the fan, decreasing the broadband noise levels. And finally the flow

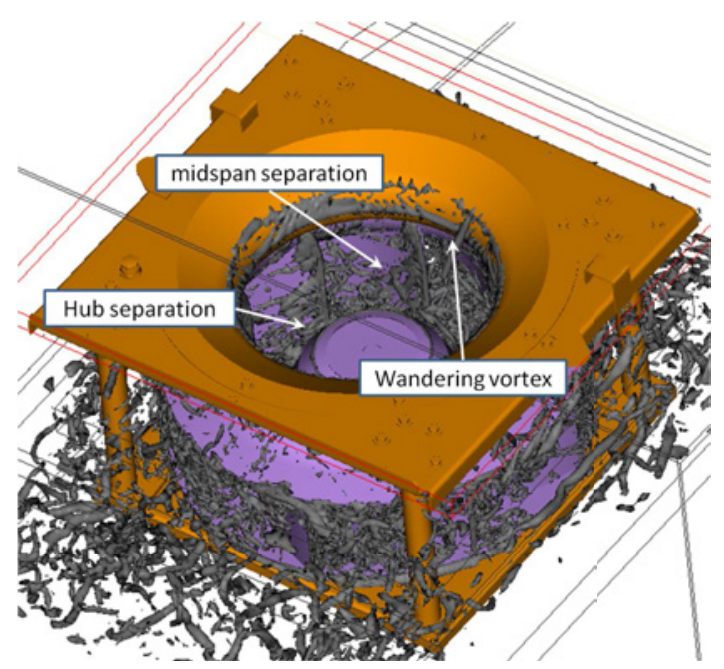

Figure 10. Iso-contours of $\lambda_{2}$ factor in the Filter simulation (filter highlighted in red).

pattern at the upper part of the blade are smoother, reducing the tone emergence.

The integrated level of the BPF tone is reduced by about $10 \mathrm{~dB}$ on the microphone M1, while the reduction is lower on microphone M2 as reported in Tab. 5. The unsteadiness of the $\mathrm{BPF}$ tone is similar as on the Baseline configuration on microphone M1, and slightly reduced on M2. Actually similar large coherent structures or "cucumber-like" vortices as shown in Fig. 3(a) are seen to wander in the hub in Fig. 10. Similar noise reduction is also observed experimentally with little modification of the BPF unsteadiness in Tab. 5. When these levels are transformed into specific noise levels to correct for the flow rate difference according to ASHRAE the maximum mean level observed on M2 becomes $72 \mathrm{~dB}$, which is still significantly lower than the Baseline case.

In summary the filter has an important effect on both the flow and the radiated sound. It avoids the large turbulent structures ingested by the fan, and improves the flow-field uniformity at the fan inlet. Although the incoming flow in the fan is improved, reducing the noise sources on the upper part 

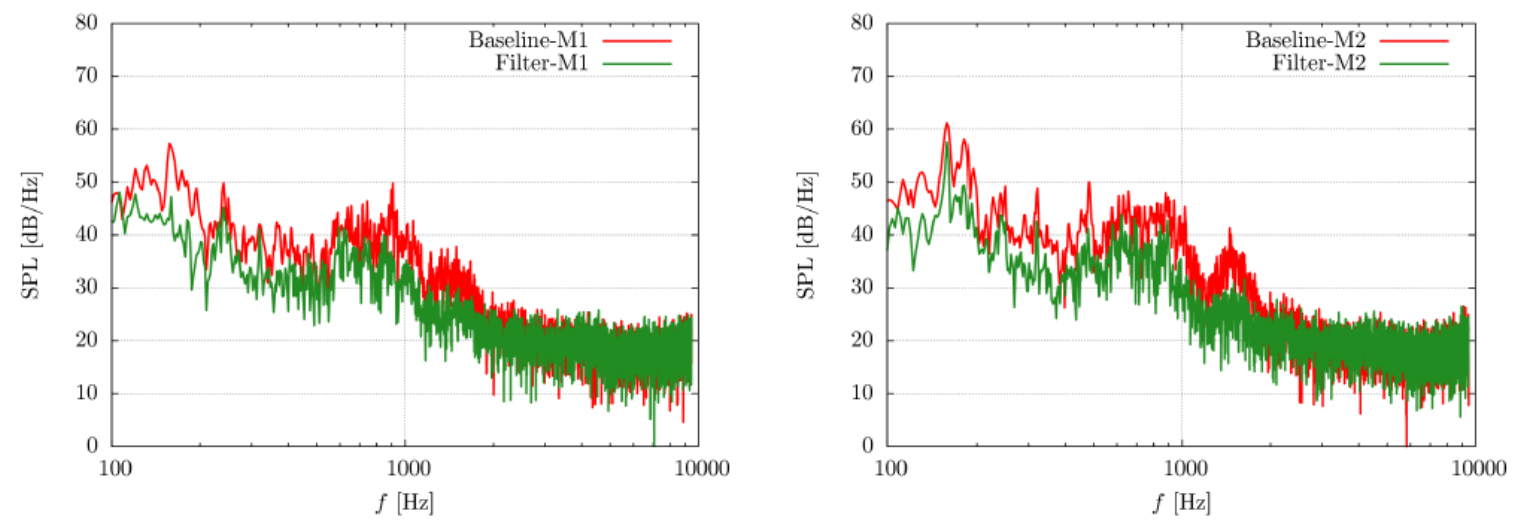

Figure 12. Filter configuration: acoustic comparisons on microphones M1 and M2.

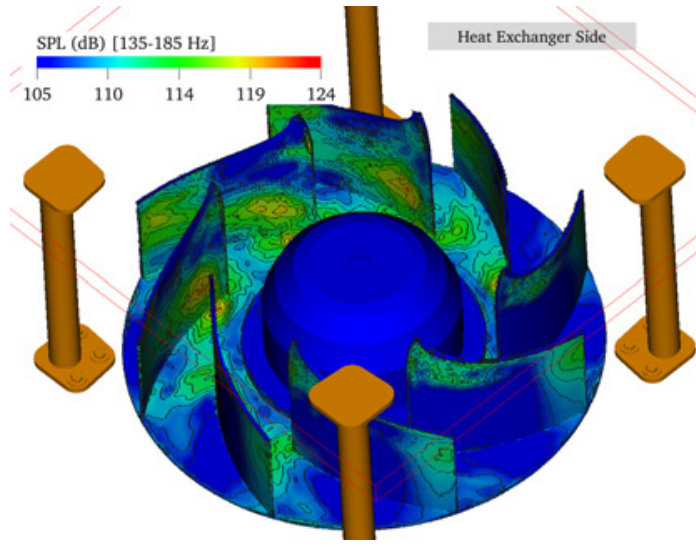

Figure 11. Band filtered power spectral density of the wall-pressure fluctuations in the Filter simulation around the $\mathrm{BPF}$ (filter highlighted in red).

Table 5. Tone peak and fluctuation amplitudes in $\mathrm{dB}$ for the Filter configuration.

\begin{tabular}{l|l|ll|l} 
Case & Mean & Mean $+\sigma$ & Mean $-\sigma$ & diff \\
\hline Exp-M1 & 66.8 & 61.9 & 69.9 & 8 \\
Exp-M2 & 70.9 & 66 & 74 & 8 \\
\hline PF-M1 & 61.7 & 54.9 & 65.4 & 10.5 \\
PF-M2 & 68.1 & 63.1 & 71.3 & 8.2
\end{tabular}

of the blades, the filter introduces an additional pressure loss yielding lower flow-rates in the mock-up. The flow angle is consequently modified at mid-height and strong flow detachments appear on the blade leading-edge. Some adaptation of the blade stacking would therefore be needed. Moreover the filter yields a reduction of the BPF unsteadiness which also suggests a possible noise control by obstructions.

\subsection{Filter+Obstruction configuration}

To achieve the final tonal noise control, a periodic flow obstruction is introduced below the filter similar to those already

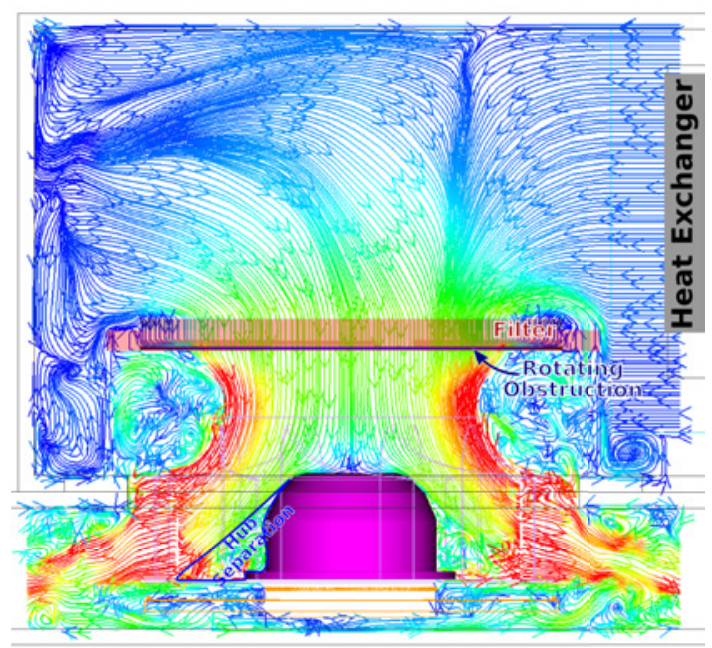

Figure 13. Filter+Obstruction configuration: flow topology (instantaneous streamlines colored by velocity magnitude; red box: filter+obstruction)).

used for centrifugal and radial fans [5, 6]. A secondary noise source is created by the impact of the wake structures (vortex rings) that appear downstream of each obstruction lobe. Because of the convergent inflow in the present configuration, these wakes are flushed with the surfaces of the bellmouth. The rotation of the flow obstruction introduces an angle in the wakes and a sound modulation that yields another tone in the far-field spectra which allows separating the primary and secondary noise sources. It also allows finding the optimum angular position of the obstruction [7]. The slight distortion induced at the blade leading edge by the obstruction wake impact has almost no effect on the relative velocity contours at the fan inlet. Surprisingly, the middle contour lines have even become axisymmetric in the Filt-Obstr simulation compared with the Filter case. Indeed the obstruction introduces an additional contraction below the filter that aligns the streamlines with the fan axis even better. Consequently the flow topology shown in Fig. 13 is similar or better in the Filt-Obstr simulation than in the Filter case. 

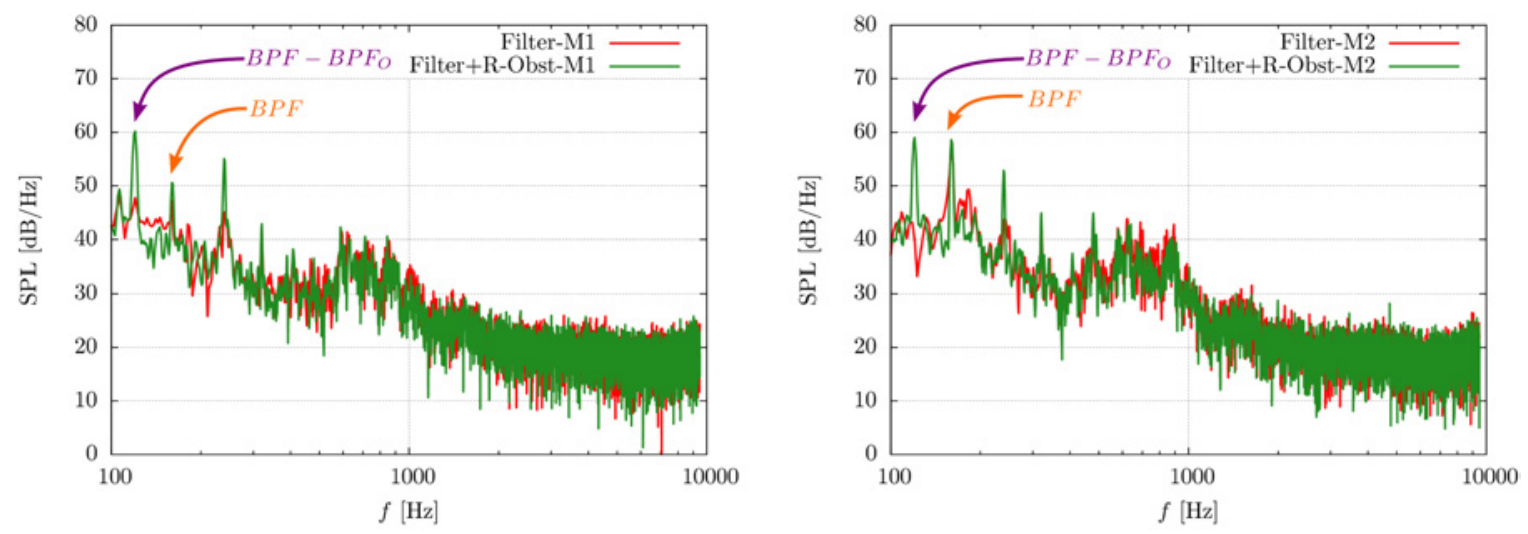

Figure 14. Filter+Obstruction configuration: acoustic comparisons on microphones M1 and M2.

The recorded far-field acoustic pressure is shown in Fig. 14. The obstruction having 8 lobes and rotating at $5 \mathrm{~Hz}$, the interaction noise tone is located at $40 \mathrm{~Hz}$ from $\mathrm{BPF}$ tone. As the obstruction and the fan rotate in the same direction, the peak appears at $120 \mathrm{~Hz}$. On microphone M2, the primary and secondary noises appear with the same amplitude, while on microphone M1, the secondary source is larger. This demonstrates a strong directivity effect. It is noteworthy to see that an additional peak at $220 \mathrm{~Hz}$ appears. This peak emerges from an already existing heap, and might be a side effect of an additional interaction, its source is not yet identified. Similar results have been obtained experimentally stressing the capability of the LBM method to simulate such a complex control configuration with a rotating obstruction.

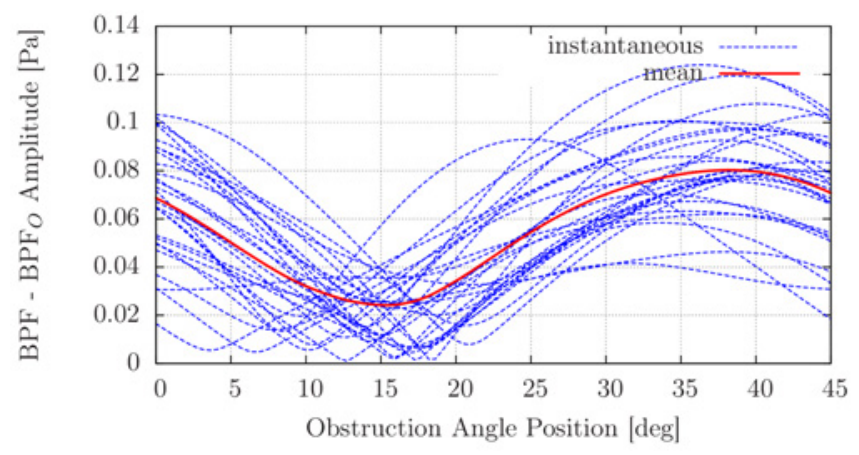

Figure 15. Attenuation as a function of the obstruction angular position. The dashed blue lines are the attenuation for several lobe passing periods on $0.8 \mathrm{~s}$ of simulated time. The red thick line is the averaged attenuation.

Following the methodology developed by Gerard et al. [8, 9] or Magne [7], a modulation effect on the tonal levels can be observed by filtering the acoustic spectra in a band containing the two primary and secondary tones. The amplitude of this band is then plotted as a function of the angular position of the obstruction for several lobe passing periods on $0.8 \mathrm{~s}$ of simulated time in Fig. 15. The best obstruction angle of maximum attenuation varies from period to period due to the BPF instabilities within the range of $25^{\circ}$ to $45^{\circ}$. Therefore, although the obstruction has an actual modulation effect on the BPF tone and an overall mean attenuation, the remaining unsteadiness of the BPF tone shown in the large spread of attenuation curves in Fig. 15 does not allow to find a fixed optimal angle for the obstruction, and still makes this passive device hardly efficient on this particular configuration. Most likely the remaining axial flow fluctuations not restrained by the thin filter are the cause of such variations. This should be actually related to the proper design of a Turbulence Control Screen or Inlet Control Device for reliable static flow and acoustic measurements of rotating machines.

\section{CONCLUSION}

The aeroacoustics of a large complex module typical of ventilation systems used in building or large transportation systems has been successfully simulated with a Lattice Boltzmann Method as implemented in Powerflow 4.4 from Exa. The available flow measurements are well captured in these simulations given the resolution achieved in the outlet ducts. Excellent agreement is achieved with all measured far-field acoustic pressure spectra, both in terms of levels and spectral shape. This is the case for both tonal and broadband noise up to $5000 \mathrm{~Hz}$, the maximum frequency that can be directly resolved by the local voxel size at the microphone location. The flow analysis has tracked the strong dominant BPF tone to be caused by the strong flow distortion at the fan inlet and possible flow separation at the blade foot caused by the hub shape. Moreover the observed strong unsteadiness of the BPF tone which experimentally prevented any noise control with an obstruction is shown to be caused by large wandering vortical structures at the fan inlet.

In order to reduce this strong tonal content several modifications have been proposed and calculated by the LBM method given its fast simulation turnaround. First the fan hub has been contoured to significantly remove the flow separation at the blade foot. Even though it improved the aerodynamic 
performances of the fan, it increased the flow distorsion at the fan inlet. Consequently the modification of the hub geometry did not bring any significant noise reduction and a satisfactory stabilisation of the BPF tone. To control the flow quality at the fan inlet a filter typical of kitchen appliances has been added on top of the fan bellmouth, and characterized experimentally to yield an equivalent porous medium form the simulations. The filter has an important effect on both the flow and the radiated sound. It avoids the large turbulent structures ingested by the fan, and improves the flow-field uniformity at the fan inlet. Consequently the tonal noise level and its unsteadiness are reduced. Similarly the broadband noise content is decreased over a large frequency range. Yet, the filter introduces an additional pressure loss yielding lower flow-rates in the mock-up which partially offset the observed large noise gains. Moreover the flow angle is consequently modified at mid-height and strong flow detachments appear on the blade leading-edge. A final tonal noise control with a rotating obstruction placed on the fan bellmouth has been succesfully simulated and tested. Even if the expected modulation and control is observed, the resulting unsteady noise attenuation still limits the control by obstructions for this particular module configuration. The excellent agreement between measurements and simulation results for all these modified configurations should be finally stressed.

\section{ACKNOWLEDGMENTS}

Computations were made on the supercomputer MammothMP2 from Université de Sherbrooke, managed by Calcul Québec and Compute Canada. The operation of this supercomputer is funded by the CFI, NanoQuébec, RMGA and FRQ-NT. The authors would like to thank F. Pérot from Exa for fruitful discussions and technical support. Finally they would like to acknowledge NSERC for funding this INNOV research project.

\section{REFERENCES}

[1] T. Carolus, M. Schneider, and H. Reese. Axial flow fan broad-band noise and prediction. Journal of sound and vibration, 300(1):50-70, 2007.

[2] F. Pérot, M.-S. Kim, S. Moreau, M. Henner, and D. Neal. Direct Aeroacoustics Prediction of a Low Speed Axial Fan. In 16th AIAA/CEAS Aeroacoustics Conference, number AIAA-2010-3887, Stockholm, Sweden, June 7-9 2010.

[3] F. Pérot, M.-S. Kim, V. Le Goff, X. Carniel, Y. Goth, and C. Chassaignon. Numerical optimization of the tonal noise of a backward centrifugal fan using a flow obstruction. Noise Control Engineering Journal, 61(3):307-319, 2013.

[4] S. Magne, M. Sanjosé, S. Moreau, and A. Berry. Aeroacoustic Prediction of the Tonal Noise Radiated by a Ring Fan in Uniform Inlet Flow. In 18th AIAA/CEAS Aeroacoustics Conference, 2012. AIAA-2012-2122.

[5] A. Gérard, M. Besombes, A. Berry, P. Masson, and S. Moreau. Tonal noise control from centrifugal fans us- ing flow control obstructions. Noise Control Engineering Journal, 61(4), 2013.

[6] M. Sanjose, S. Moreau, and J. Gullbrand. Hybrid Noise Predictions Of A Radial Notebook Blower. In $16^{\text {th }}$ International Symposium on Transport Phenomena and Dynamics of Rotating Machinery, Honolulu, HI, USA, April 2016.

[7] S. Magne. Approche numérique du contrôle du bruit tonal des ventilateurs par obstruction de l'écoulement. $\mathrm{PhD}$ thesis, Université de Sherbrooke, 2015.

[8] A. Gérard, A.Berry, P. Masson, and S. Moreau. Use of a beat effect for the automatic positioning of flow obstructions to control tonal fan noise: Theory and experiments. Journal of Sound and Vibration, 332(19):44504460, 2013.

[9] A. Gérard, S. Moreau, A. Berry, and P. Masson. Design of multi-modal obstruction to control tonal fan noise using modulation principles. Journal of Sound and Vibration, 356:34-47, 2015. 